\title{
Vieta's Formula about the Sum of Roots of Polynomials
}

\author{
Artur Korniłowicz \\ Institute of Informatics \\ University of Białystok \\ Poland
}

\author{
Karol Pąk \\ Institute of Informatics \\ University of Białystok \\ Poland
}

Summary. In the article we formalized in the Mizar system [2] the Vieta formula about the sum of roots of a polynomial $a_{n} x^{n}+a_{n-1} x^{n-1}+\cdots+a_{1} x+a_{0}$ defined over an algebraically closed field. The formula says that $x_{1}+x_{2}+\cdots+$ $x_{n-1}+x_{n}=-\frac{a_{n-1}}{a_{n}}$, where $x_{1}, x_{2}, \ldots, x_{n}$ are (not necessarily distinct) roots of the polynomial [12]. In the article the sum is denoted by SumRoots.

MSC: 12E05 03B35

Keywords: roots of polynomials; Vieta's formula

MML identifier: POLYVIE1, version: 8.1.06 5.43.1297

Let $F$ be a finite sequence and $f$ be a function $\operatorname{from} \operatorname{dom} F \operatorname{into} \operatorname{dom} F$. Observe that $F \cdot f$ is finite sequence-like.

Now we state the propositions:

(1) Let us consider objects $a, b$. Suppose $a \neq b$. Then

(i) $\operatorname{CFS}(\{a, b\})=\langle a, b\rangle$, or

(ii) $\operatorname{CFS}(\{a, b\})=\langle b, a\rangle$.

(2) Let us consider a finite set $X$. Then $\operatorname{CFS}(X)$ is an enumeration of $X$.

Let $A$ be a set and $X$ be a finite subset of $A$. Observe that $\operatorname{CFS}(X)$ is $A$-valued.

Now we state the proposition:

(3) Let us consider a right zeroed, non empty additive loop structure $L$, and an element $a$ of $L$. Then $2 \cdot a=a+a$. 
Let $L$ be an almost left invertible multiplicative loop with zero structure. Let us note that every element of $L$ which is non zero is also left invertible.

Let $L$ be an almost right invertible multiplicative loop with zero structure. Observe that every element of $L$ which is non zero is also right invertible.

Let $L$ be an almost left cancelable multiplicative loop with zero structure. Let us observe that every element of $L$ which is non zero is also left mult-cancelable.

Let $L$ be an almost right cancelable multiplicative loop with zero structure. One can verify that every element of $L$ which is non zero is also right multcancelable.

Now we state the proposition:

(4) Let us consider a right unital, associative, non trivial double loop structure $L$, and elements $a, b$ of $L$. Suppose $b$ is left invertible and right multcancelable and $b \cdot \frac{1}{b}=\frac{1}{b} \cdot b$. Then $\frac{a \cdot b}{b}=a$.

Let $L$ be a non degenerated zero-one structure, $z_{0}$ be an element of $L$, and $z_{1}$ be a non zero element of $L$. Note that $\left\langle z_{0}, z_{1}\right\rangle$ is non-zero and $\left\langle z_{1}, z_{0}\right\rangle$ is non-zero.

Let us consider a non trivial zero structure $L$ and a polynomial $p$ over $L$. Now we state the propositions:

(5) If len $p=1$, then there exists a non zero element $a$ of $L$ such that $p=\langle a\rangle$.

(6) If len $p=2$, then there exists an element $a$ of $L$ and there exists a non zero element $b$ of $L$ such that $p=\langle a, b\rangle$.

(7) If len $p=3$, then there exist elements $a, b$ of $L$ and there exists a non zero element $c$ of $L$ such that $p=\langle a, b, c\rangle$.

Now we state the propositions:

(8) Let us consider an add-associative, right zeroed, right complementable, associative, commutative, left distributive, well unital, almost left invertible, non empty double loop structure $L$, and elements $a, b, x$ of $L$. If $b \neq 0_{L}$, then $\operatorname{eval}\left(\langle a, b\rangle,-\frac{a}{b}\right)=0_{L}$.

(9) Let us consider a field $L$, elements $a, x$ of $L$, and a non zero element $b$ of $L$. Then $x$ is a root of $\langle a, b\rangle$ if and only if $x=-\frac{a}{b}$. The theorem is a consequence of (4) and (8).

Let us consider a field $L$, an element $a$ of $L$, and a non zero element $b$ of $L$. Now we state the propositions:

(10) $\operatorname{Roots}(\langle a, b\rangle)=\left\{-\frac{a}{b}\right\}$. The theorem is a consequence of (9).

(11) multiplicity $\left(\langle a, b\rangle,-\frac{a}{b}\right)=1$. The theorem is a consequence of (9).

(12) $\operatorname{BRoots}(\langle a, b\rangle)=\left(\left\{-\frac{a}{b}\right\}, 1\right)$-bag. The theorem is a consequence of (10) and (11).

(13) Let us consider a field $L$, elements $a, c$ of $L$, and non zero elements $b, d$ of $L$. Then $\operatorname{Roots}(\langle a, b\rangle *\langle c, d\rangle)=\left\{-\frac{a}{b},-\frac{c}{d}\right\}$. The theorem is a consequence 
of $(10)$.

(14) Let us consider a field $L$, elements $a, x$ of $L$, and a non zero element $b$ of $L$. If $x \neq-\frac{a}{b}$, then multiplicity $(\langle a, b\rangle, x)=0$. The theorem is a consequence of $(10)$.

Let us consider a field $L$, a non-zero polynomial $p$ over $L$, an element $a$ of $L$, and a non zero element $b$ of $L$. Now we state the propositions:

(15) Suppose $-\frac{a}{b} \notin \operatorname{Roots}(p)$. Then $\overline{\overline{\operatorname{Roots}(\langle a, b\rangle * p)}}=1+\overline{\overline{\operatorname{Roots}(p)}}$. The theorem is a consequence of (10).

(16) Suppose $-\frac{a}{b} \notin \operatorname{Roots}(p)$. Then $\operatorname{CFS}(\operatorname{Roots}(p))^{\frown}\left\langle-\frac{a}{b}\right\rangle$ is an enumeration of $\operatorname{Roots}(\langle a, b\rangle * p)$. The theorem is a consequence of (10).

(17) Let us consider a field $L$, a non-zero polynomial $p$ over $L$, an element $a$ of $L$, a non zero element $b$ of $L$, and an enumeration $E$ of $\operatorname{Roots}(\langle a, b\rangle * p)$. Suppose $E=\operatorname{CFS}(\operatorname{Roots}(p)) \frown\left\langle-\frac{a}{b}\right\rangle$. Then

(i) len $E=1+\overline{\overline{\operatorname{Roots}(p)}}$, and

(ii) $E(1+\overline{\overline{\operatorname{Roots}(p)}})=-\frac{a}{b}$, and

(iii) for every natural number $n$ such that $1 \leqslant n \leqslant \overline{\overline{\operatorname{Roots}(p)}}$ holds $E(n)=$ $(\operatorname{CFS}(\operatorname{Roots}(p)))(n)$.

Let $L$ be a non empty double loop structure, $B$ be a bag of the carrier of $L$, and $E$ be a (the carrier of $L$ )-valued finite sequence. The functor $B(++) E$ yielding a finite sequence of elements of $L$ is defined by

(Def. 1) len $i t=$ len $E$ and for every natural number $n$ such that $1 \leqslant n \leqslant$ len it holds $i t(n)=(B \cdot E)(n) \cdot E_{n}$.

Now we state the propositions:

(18) Let us consider an integral domain $L$, a non-zero polynomial $p$ over $L$, a bag $B$ of the carrier of $L$, and an enumeration $E$ of $\operatorname{Roots}(p)$. If $\operatorname{Roots}(p)=\emptyset$, then $B(++) E=\emptyset$.

(19) Let us consider a left zeroed, add-associative, non empty double loop structure $L$, bags $B_{1}, B_{2}$ of the carrier of $L$, and a (the carrier of $L$ )-valued finite sequence $E$. Then $B_{1}+B_{2}(++) E=\left(B_{1}(++) E\right)+\left(B_{2}(++) E\right)$.

(20) Let us consider a left zeroed, add-associative, non empty double loop structure $L$, a bag $B$ of the carrier of $L$, and (the carrier of $L$ )-valued finite sequences $E, F$. Then $B(++) E^{\frown} F=(B(++) E)^{\frown}(B(++) F)$.

(21) Let us consider a left zeroed, add-associative, non empty double loop structure $L$, bags $B_{1}, B_{2}$ of the carrier of $L$, and (the carrier of $L$ )valued finite sequences $E, F$. Then $B_{1}+B_{2}(++) E \frown F=\left(B_{1}(++) E\right) \frown$ $\left(B_{1}(++) F\right)+\left(B_{2}(++) E\right)^{\frown}\left(B_{2}(++) F\right)$. The theorem is a consequence of (19) and (20). 
(22) Let us consider a field $L$, a non-zero polynomial $p$ over $L$, an element $a$ of $L$, a non zero element $b$ of $L$, an enumeration $E$ of $\operatorname{Roots}(\langle a, b\rangle * p)$, and a permutation $P$ of $\operatorname{dom} E$. Then $(\operatorname{BRoots}(\langle a, b\rangle * p)(++) E) \cdot P=$ $\operatorname{BRoots}(\langle a, b\rangle * p)(++)(E \cdot P)$.

Proof: Set $q=\langle a, b\rangle$. Set $B=\operatorname{BRoots}(q * p)$. Reconsider $P_{1}=P$ as a permutation of $\operatorname{dom}(B(++) E) \cdot(B(++) E) \cdot P_{1}=B(++)(E \cdot P)$ by [13, (27)], [11, (29), (25)], [4, (13)].

Let us consider a field $L$, a non-zero polynomial $p$ over $L$, an element $a$ of $L$, a non zero element $b$ of $L$, and an enumeration $E$ of $\operatorname{Roots}(\langle a, b\rangle * p)$. Now we state the propositions:

(23) Suppose $-\frac{a}{b} \notin \operatorname{Roots}(p)$. Then suppose $E=\operatorname{CFS}(\operatorname{Roots}(p))^{\frown}\left\langle-\frac{a}{b}\right\rangle$. Then $(\operatorname{CFS}(\operatorname{Roots}(\langle a, b\rangle * p)))^{-1} \cdot E$ is a permutation of $\operatorname{dom} E$. The theorem is a consequence of (15) and (10).

(24) Suppose $-\frac{a}{b} \notin \operatorname{Roots}(p)$. Then suppose $E=\operatorname{CFS}(\operatorname{Roots}(p))^{\wedge}\left\langle-\frac{a}{b}\right\rangle$. Then $\sum(\operatorname{BRoots}(\langle a, b\rangle * p)(++) E)=\sum(\operatorname{BRoots}(\langle a, b\rangle * p)(++) \operatorname{CFS}(\operatorname{Roots}(\langle a, b\rangle *$ $p))$ ).

Proof: Set $q=\langle a, b\rangle$. Set $B=\operatorname{BRoots}(q * p)$. Set $D=\operatorname{CFS}(\operatorname{Roots}(q * p))$. Reconsider $P=D^{-1} \cdot E$ as a permutation of $\operatorname{dom} E \cdot E \cdot E^{-1} \cdot D=D$ by [4, (37)], [13, (27)], [4, (35), (12)]. $(B(++) E) \cdot P^{-1}=B(++)\left(E \cdot P^{-1}\right)$.

(25) $\sum(\operatorname{BRoots}(\langle a, b\rangle)(++) E)=-\frac{a}{b}$. The theorem is a consequence of (10), (11), and (14).

Let $L$ be an integral domain and $p$ be a non-zero polynomial over $L$. The functor $\operatorname{SumRoots}(p)$ yielding an element of $L$ is defined by the term

(Def. 2) $\sum(\operatorname{BRoots}(p)(++) \operatorname{CFS}(\operatorname{Roots}(p)))$.

Now we state the propositions:

(26) Let us consider an integral domain $L$, and a non-zero polynomial $p$ over $L$. If $\operatorname{Roots}(p)=\emptyset$, then $\operatorname{SumRoots}(p)=0_{L}$. The theorem is a consequence of (2) and (18).

(27) Let us consider a field $L$, an element $a$ of $L$, and a non zero element $b$ of $L$. Then $\operatorname{SumRoots}(\langle a, b\rangle)=-\frac{a}{b}$. The theorem is a consequence of (10), (2), and (11).

(28) Let us consider a field $L$, a non-zero polynomial $p$ over $L$, an element $a$ of $L$, and a non zero element $b$ of $L$. Then $\operatorname{SumRoots}(\langle a, b\rangle * p)=$ $-\frac{a}{b}+\operatorname{SumRoots}(p)$. The theorem is a consequence of (16), (17), (24), (2), (10), (11), (25), and (19).

(29) Let us consider a field $L$, elements $a, c$ of $L$, and non zero elements $b, d$ of $L$. Then $\operatorname{SumRoots}(\langle a, b\rangle *\langle c, d\rangle)=-\frac{a}{b}+-\frac{c}{d}$. The theorem is a consequence of (27) and (28). 
(30) Let us consider an algebraic closed field $L$, and non-zero polynomials $p, q$ over $L$. Suppose len $p \geqslant 2$. Then $\operatorname{SumRoots}(p * q)=\operatorname{SumRoots}(p)+$ $\operatorname{SumRoots}(q)$.

Proof: Define $\mathcal{P}$ [natural number] $\equiv$ for every non-zero polynomial $f$ over $L$ such that $\$_{1}=$ len $f$ holds $\operatorname{SumRoots}(f * q)=\operatorname{SumRoots}(f)+$ $\operatorname{SumRoots}(q) \cdot \mathcal{P}[2]$. For every non trivial natural number $k$ such that $\mathcal{P}[k]$ holds $\mathcal{P}[k+1]$ by [6, (29)], [1, (11)], [8, (17), (50)]. For every non trivial natural number $k, \mathcal{P}[k]$ from [6, Sch. 2].

(31) Let us consider an algebraic closed integral domain $L$, a non-zero polynomial $p$ over $L$, and a finite sequence $r$ of elements of $L$. Suppose $r$ is one-toone and len $r=\operatorname{len} p-{ }^{\prime} 1$ and $\operatorname{Roots}(p)=\operatorname{rng} r$. Then $\sum r=\operatorname{SumRoots}(p)$. Proof: Set $B=\operatorname{BRoots}(p)$. Set $s=\operatorname{support} B$. Set $L_{1}=\operatorname{len} r \mapsto 1$. Consider $f$ being a finite sequence of elements of $\mathbb{N}$ such that degree $(B)=$ $\sum f$ and $f=B \cdot \operatorname{CFS}(s)$. Reconsider $E=\operatorname{CFS}(s)$ as a finite sequence of elements of $L$. For every natural number $j$ such that $j \in \operatorname{Seg} \operatorname{len} r$ holds $f(j) \geqslant L_{1}(j)$ by [8, (52)], [4, (12)], [3, (57)]. For every natural number $j$ such that $1 \leqslant j \leqslant$ len $E$ holds $(B(++) E)(j)=E(j)$ by [5, (83)], [3. (57)], [9, (13)].

(32) VietA's Formula ABout the sum of RoOts:

Let us consider an algebraic closed field $L$, and a non-zero polynomial $p$ over $L$. Suppose len $p \geqslant 2$. Then $\operatorname{SumRoots}(p)=-\frac{p\left(\operatorname{len} p-^{\prime} 2\right)}{p\left(\operatorname{len} p-^{\prime} 1\right)}$.

Proof: Define $\mathcal{P}$ [natural number] $\equiv$ for every non-zero polynomial $p$ over $L$ such that $\$_{1}=$ len $p$ holds $\operatorname{SumRoots}(p)=-\frac{p\left(\$_{1}{ }^{\prime} 2\right)}{p\left(\$_{1}-^{\prime} 1\right)} \cdot \mathcal{P}[2]$ by $(6),[7$, (38)], (27). For every non trivial natural number $k$ such that $\mathcal{P}[k]$ holds $\mathcal{P}[k+1]$ by [6, (29)], [1, (11)], [8, (17)], [10, (5)]. For every non trivial natural number $k, \mathcal{P}[k]$ from [6, Sch. 2].

\section{REFERENCES}

[1] Grzegorz Bancerek. The fundamental properties of natural numbers Formalized Mathematics, 1(1):41-46, 1990.

[2] Grzegorz Bancerek, Czesław Byliński, Adam Grabowski, Artur Korniłowicz, Roman Matuszewski, Adam Naumowicz, Karol Pąk, and Josef Urban. Mizar: State-of-the-art and beyond. In Manfred Kerber, Jacques Carette, Cezary Kaliszyk, Florian Rabe, and Volker Sorge, editors, Intelligent Computer Mathematics, volume 9150 of Lecture Notes in Computer Science, pages 261-279. Springer International Publishing, 2015. ISBN 978-3319-20614-1. doi 10.1007/978-3-319-20615-8_17.

[3] Czesław Byliński. Finite sequences and tuples of elements of a non-empty sets. Formalized Mathematics, 1(3):529-536, 1990 .

[4] Czesław Byliński. Functions and their basic properties. Formalized Mathematics, 1(1): 55-65, 1990.

[5] Czesław Byliński. The sum and product of finite sequences of real numbers Formalized Mathematics, 1(4):661-668, 1990.

[6] Robert Milewski. Natural numbers. Formalized Mathematics, 7(1):19-22, 1998. 
[7] Robert Milewski. Fundamental theorem of algebra Formalized Mathematics, 9(3):461470, 2001.

[8] Piotr Rudnicki. Little Bezout theorem (factor theorem) Formalized Mathematics, 12(1): 49-58, 2004.

[9] Christoph Schwarzweller. The binomial theorem for algebraic structures Formalized Mathematics, 9(3):559-564, 2001.

[10] Michał J. Trybulec. Integers Formalized Mathematics, 1(3):501-505, 1990.

[11] Wojciech A. Trybulec. Non-contiguous substrings and one-to-one finite sequences. Formalized Mathematics, 1(3):569-573, 1990.

[12] E. B. Vinberg. A Course in Algebra. American Mathematical Society, 2003. ISBN 0821834134.

[13] Edmund Woronowicz. Relations and their basic properties Formalized Mathematics, 1 (1):73-83, 1990.

Received May 25, 2017

The English version of this volume of Formalized Mathematics was financed under agreement 548/P-DUN/2016 with the funds from the Polish Minister of Science and Higher Education for the dissemination of science. 